\title{
Numerical Modeling of PCB Power/Ground Plate-Pairs by DGTD Method Taking Into Account Decoupling Capacitors
}

\author{
Ping Li and Li Jun Jiang \\ Department of Electrical and Electronic Engineering The University of Hong Kong Hong Kong \\ Email: liping@eee.hku.hk, jianglj@hku.hk \\ Hakan Bağc1 \\ Division of Computer, Electrical, and Mathematical Sciences and Engineering King Abdullah University of \\ Science and Technology, Thuwal, 23955-6900, Saudi Arabia \\ Email: hakan.bagci@kaust.edu.sa
}

(Invited Paper)

\begin{abstract}
A discontinuous Galerkin time-domain (DGTD) method is proposed in this work to analyze printed circuit board (PCB) power/ground plate-pair having arbitrarily shaped antipads. To apply proper excitation source over the irregular antipad, the implemented wave port magnetic current excitation is expanded by the electric eigen-modes of the anti-pad that are calculated via either numerical approach or analytical method. Based on the orthogonality of eigen-modes, the temporal mode expansion coefficient for each mode can be conveniently extracted. Besides, considering the presence of decoupling capacitors, the whole physical system can be split into field and circuit subsystems. For the field subsystem, it is governed by the Maxwell's equations, thus it will be solved by DGTD method. For the circuit subsystem, the modified nodal analysis (MNA) is applied. In order to achieve the coupling between the field and circuit subsystems, a lumpled port is defined at the interface between the field and circuit subsystems. To verify the proposed algorithm, several representative examples are benchmarked.
\end{abstract}

\section{INTRODUCTION}

Vias crossing the power/ground plate-pairs not only provide the routine for the DC voltage supply, but also serve as the return path for the high-frequency signals. Thereby, parasitic noise currents might be induced in the nearby power/ground vias, which will cause voltage fluctuation over the power deliver networks (PDN). A well-designed multilayer power/ground plane should have the capability to suppress the noise and thus main both signal and power integrities. Therefore, comprehensive and rigorous analysis of power/groound plate pairs is necessary. To reach this aim, a large number of treatments have been proposed over the past years such as the general scattering method based on the Foldy-Lax scattering equation and mode expansion of Green's function [1], the hybrid finite-element method (FEM) [2], and the equivalent circuit models [3].

In this work, a discontinuous Galerkin time-domain (DGTD) [4] method is developed to analyze the multilayer powerground plate-pairs. DGTD integrates the advantages of finite volume method (FVM) and FEM, thus it can achieve both locality and high-order accuracy. To enforce proper wave port excitation over the anti-pad, the magnetic current source used
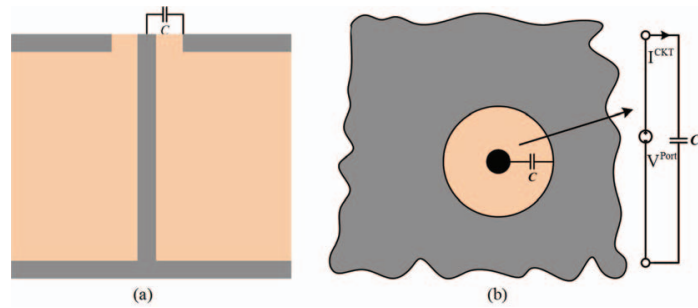

Fig. 1. Schematic illustration of the circular lumped port where the fieldcircuit coupling happens. (a) A decoupling capacitor $C$ is added between the power-ground planes through a metal via. (b) To model the capacitor, a circular lumped port is defined.

in this work is expanded by the eigen-modes supported by the anti-pad. In this way, only the modes of interests can be used for excitation. By leveraging the orthogonal property of eigen-modes, the incident, transmitted and reflected mode expansion coefficients in the time-domain can be accurately extracted. Then, the S-parameters are able to be obtained based on the pre-calculated mode coefficients. Furthermore, lumped capacitors are broadly used as to block the high frequency noise. To incorporate the decoupling capacitor into the DGTD analysis, the computational system can be decomposed into field and circuit subsystem. For the circuit subsystem, the modified nodal analysis (MNA) algorithm is used to construct the circuit equations; while for the field subsystem, the DGTD method is applied to solve the two first-order time-domain Maxwell's equations. To achieve the mutual coupling between the EM and circuit susyems, a coaxial lumped port is defined over which a lumped voltage source is introduced to achieve the coupling from the EM to the circuit systems and a lumped port current is employed to facilitate the coupling from the circuit to the EM system. Based on this coupling scheme, a hybrid field-circuit matrix equation can be established, and which can be further solved in an synchronous timemarching scheme. Thus, both the instability and the accuracy are guaranteed. 


\section{FORMULATION}

Decoupling capacitors are placed between the power and ground planes to immediately provide the transient currents to the switching transistors, for general decoupling networks, the MNA approach can be employed to establish the circuit equations regardless of the complexity. In order to model the lumped capacitor $C$ as well as its parasitic components including an equivalent series inductor (ESL) $L$ and an equivalent series resistor (ESR) $R$, a circular lumped port shown in Fig. 1 (b) is introduced. At the lumped port, a lumped surface current density $\mathbf{J}^{\mathrm{CKT}}(\rho, \phi)$ flowing along the radial direction $\hat{\boldsymbol{\rho}}$ is introduced to achieve the coupling from the capacitor to the field solver. Specifically, the surface current serves as an impressed current source and thus it can be added to the Maxwell-Ampere's law equation. Simultaneously, to facilitate the coupling from the field to the decoupling capacitor, a lumped port voltage $V_{\text {port }}$ is defined, which is calculated from the line integral of the electric field along the radial direction. Namely, at time $t=(n+1) \delta t$,

$$
\mathrm{V}^{\text {port }}=-\int \mathbf{E}_{\rho} \cdot \hat{\boldsymbol{\rho}} d \rho
$$

Having the voltage given by (1), the current $\mathrm{I}^{\mathrm{CKT}}$ flowing through the capacitor can be conveniently calculated according to the following MNA equations. Namely,

$$
\left[\begin{array}{cc}
{[\mathbf{G}]} & {\left[\mathbf{B}_{1}\right]} \\
{\left[\mathbf{B}_{2}\right]} & {[0]}
\end{array}\right]\left[\begin{array}{c}
\mathbf{V}_{n+1}^{\mathrm{CKT}} \\
\mathbf{I}_{n+1}^{\mathrm{CKT}}
\end{array}\right]=\left[\begin{array}{c}
\mathbf{I}_{n}^{\mathrm{CP}} \\
\mathbf{V}_{n+1}^{\text {Port }}
\end{array}\right]
$$

Where the admittance matrix $[\mathbf{G}]$ is determined by interconnections between circuit components.

On the other side, to achieve the coupling from the circuit to the field, a surface current source $\mathbf{J}^{\mathrm{CKT}}$ over the lumped port is introduced.

By conducting the discontinuous Galerkin testing over the two first-order derivative Maxwell's equations, and approximating the first order time-derivative by central difference method, two discrete matrix equations can be obtained as

$$
\begin{gathered}
\overline{\mathbf{M}}_{e}^{i} \mathbf{e}_{n+1}^{i}+\Delta t \frac{\mathbf{j}_{e, n+1}^{i}}{2}=\overline{\mathbf{M}}_{e}^{i} \mathbf{e}_{n}^{i}+\Delta t\left[\overline{\mathbf{S}}_{e}^{i} \mathbf{h}_{n+\frac{1}{2}}^{i}-\frac{\mathbf{j}_{e, n}^{i}}{2}\right. \\
\left.+\overline{\mathbf{F}}_{e e}^{i i} \mathbf{e}_{n}^{i}-\overline{\mathbf{F}}_{e e}^{i j} \mathbf{e}_{n}^{j}-\overline{\mathbf{F}}_{e h}^{i i} \mathbf{h}_{n+\frac{1}{2}}^{i}+\overline{\mathbf{F}}_{e h}^{i j} \mathbf{h}_{n+\frac{1}{2}}^{j}\right] \\
\overline{\mathbf{M}}_{h}^{i} \mathbf{h}_{n+\frac{3}{2}}^{i}=\overline{\mathbf{M}}_{h}^{i} \mathbf{h}_{n+\frac{1}{2}}^{i}-\Delta t\left[\overline{\mathbf{S}}_{h}^{i} \mathbf{e}_{n+1}^{i}-\mathbf{j}_{h, n+1}^{i}-\overline{\mathbf{F}}_{h h}^{i i} \mathbf{h}_{n+\frac{1}{2}}^{i}\right. \\
\left.+\overline{\mathbf{F}}_{h h}^{i j} \mathbf{h}_{n+\frac{1}{2}}^{j}-\overline{\mathbf{F}}_{h e}^{i i} \mathbf{e}_{n+1}^{i}+\overline{\mathbf{F}}_{h e}^{i j} \mathbf{e}_{n+1}^{j}\right]
\end{gathered}
$$

In light of the layered property of the power-ground platepairs, in this work, triangular prism elements [4] are employed as the basic mesh cells. The finalized 3-D triangular prism elements are obtained by extending the 2-D triangles along the growing direction of the plate-pairs.

In order to main the stability of the hybrid system, a synchronous time-marching scheme is employed, which means we have to construct a circuit-field coupling matrix equation. To reach this purpose, we integrate the circuit equation in (2)

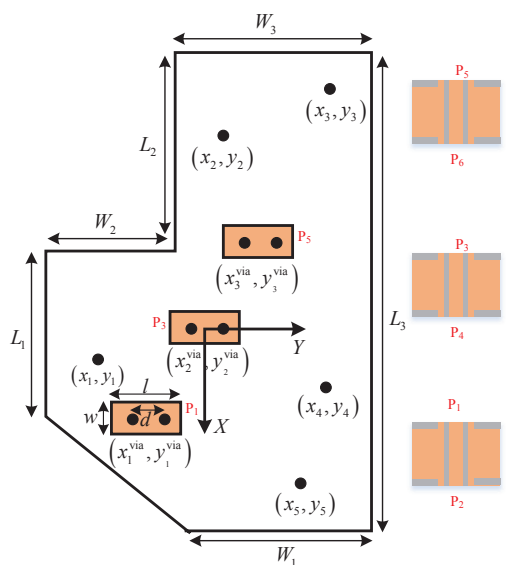

Fig. 2. Geometrical illustration of the irregular parallel plate pair with three differential via-pairs having rectangular anti-pads. The separation distance $d$ of the differential pair is $0.508 \mathrm{~mm}$, the width $w$ and length $l$ of the anti-pad are $0.762 \mathrm{~mm}$ and $1.27 \mathrm{~mm}$, respectively. The radius of the PEC rods $r_{r}$ is $0.127 \mathrm{~mm}$, the radius of the via barrel $r_{b}$ is $0.127 \mathrm{~mm}$.

with the fully-discrete Ampere's law equation in (3), and then a coupling matrix equation can be formulated as

$$
\mathbf{F}_{i}\left(\mathbf{x}_{n+1}\right)=\mathbf{b}_{n}^{i}
$$

where

$$
\mathbf{F}_{i}\left(\mathbf{x}_{n+1, i}\right)=\left[\begin{array}{ccc}
{\left[\mathbf{M}_{e}^{i}\right]} & \mathbf{0} & \delta t\left[\mathrm{j}_{e}^{i} / 2\right] \\
\mathbf{0} & {[\mathbf{G}]} & {\left[\mathbf{B}_{1}\right]} \\
{\left[\mathbf{C}^{i}\right]} & -\left[\mathbf{B}_{2}\right] & -[\mathbf{0}]
\end{array}\right]\left[\begin{array}{c}
\mathbf{e}_{n+1}^{(i)} \\
\mathbf{V}_{n+1}^{\mathrm{CKT}} \\
\mathbf{I}_{n+1}^{\mathrm{CKT}}
\end{array}\right]
$$

In this way, the field and circuit equations can be solved together at the same time, thus instability issues are avoided. Due to the local operation of DGTD, the established coupling matrix is also local and therefore its dimension is equal to the number of degree of freedom (DOF) of electric field (denoted by $n_{e}^{i}$ ) in the mesh element $i$ plus the number of the circuit unknowns (denoted by $n^{\mathrm{CKT}}$ ).

\section{NUMERICAL RESUlTS}

\section{A. A Powerr/Ground Plate Pair with Differential-Vias}

In this example, an irregular plate-pair as shown in Fig. 2 including three differential via-pairs with rectangular antipads is benchmarked. For this example, the magnetic current excitations required electric eigen-fields for common-mode is numerically calculated in advance. The power/ground platepair has four layers, and each layer has same thickness $h_{i}=0.254 \mathrm{~mm}, i=1, \cdots, 4$. In Fig. 3 and 4 , the common-mode S-parameters for port-pairs 1 and 2, 5 and 6 are shown. To validate the accuracy of the proposed algorithm, the results from HFSS simulation are given. It is noted that good agreements are achieved.

\section{B. A Power/Groud Pate Pair with Decoupling Capacitors}

To validate the algorithm for the presence of decoupling capacitors, an irregular power-ground plate-pair shown in Fig. 5 is investigated, where the two antipads are designed 


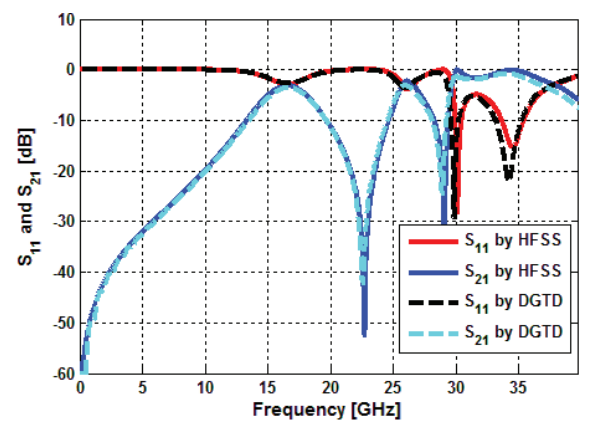

(a)

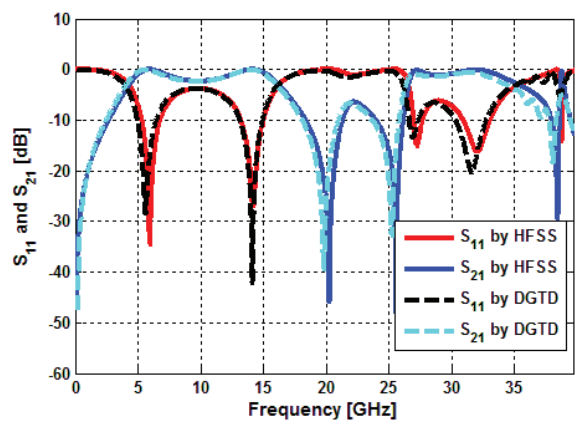

(b)

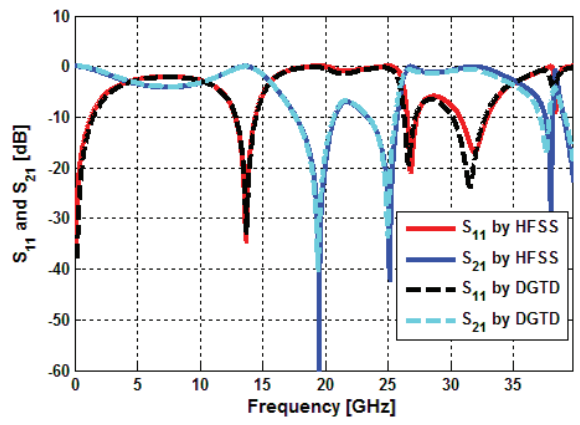

(c)

Fig. 6. Extracted S-parameters for (a) ideal decoupling capacitors, (b) non-ideal decoupling capacitors with ESRL, and (c) in the absence of decoupling capacitors.

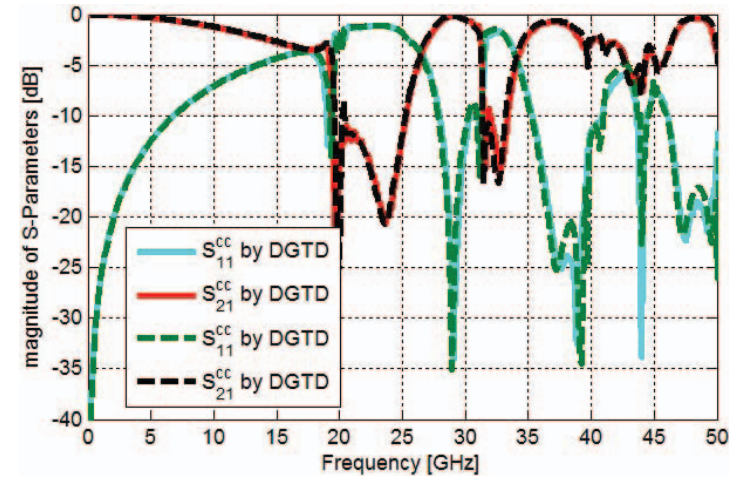

Fig. 3. The calculated common-mode S-parameters of port-pair 1 and 2 for four layer plate-pair in Fig. 2 filled with nondispersive media and the results from HFSS.

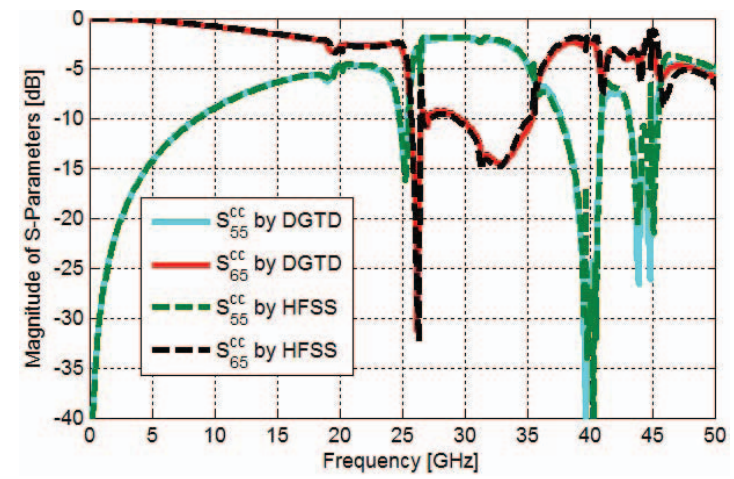

Fig. 4. The calculated common-mode S-parameters of port-pair 5 and 6 for four layer plate-pair in Fig. 2 filled with nondispersive media and the results from HFSS.

as polyhedrons with eight edges. To find out the TEM eigenelectric field supported by this kind of antipad, the Laplace equation is firstly solved to find the potential distribution. The S-parameters are evaluated according to the extracted mode coefficients, as shown in Fig. 6. As can be seen, most of the signals located in the high-frequency band expect the DC are bypassed by the decoupling capacitors. Thus, only the DC supply will be delivered by the PDN. For comparison, the results from HFSS are also given.

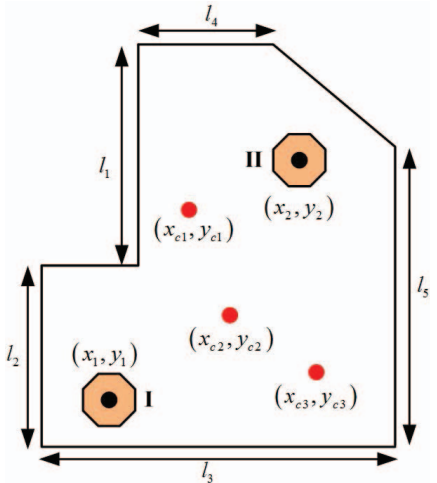

Fig. 5. Geometrical illustration of the power/ground pair with three decoupling capacitors (denoted by the red dots) and two antipads, where $l_{1}=3.81$ $\mathrm{mm}, l_{2}=3.81 \mathrm{~mm}, l_{3}=5.08 \mathrm{~mm}, l_{4}=1 \mathrm{~mm}, l_{5}=5.715 \mathrm{~mm}$, and the thickness of the power/ground pair is $h=0.254 \mathrm{~mm}$.

\section{CONCLUSION}

In this work, a DGTD algorithm is proposed for the analysis of power-ground planes with arbitrarily-shaped antipads. To implement the proper excitation, the wave port is employed. Furthermore, to consider the presence of the decoupling capacitors, a hybrid field-circuit solver is proposed. Two representative examples are benchmarked to verify the algorithm.

\section{REFERENCES}

[1] L. Tsang, H. Chen, C. C. Huang, and V. Jandhyala, "Modeling of multiple scattering among vias in planar waveguides using Foldy-Lax equations," Microw. Opt. Technol. Lett., vol. 31, pp. 201-208, Nov. 2001.

[2] Y. J. Zhang, L. H. Ren, D. Z. Liu, S. De, X. X. Gu, Y. H. Kwark, C. Schuster, and J. Fan, "An efficient hybrid finite-element analysis of multiple vias sharing the same anti-pad in an arbitrarily shaped parallelplate pair," IEEE Trans. Microw. Theory Tech., vol. 63, no. 3, pp. 883-890, Mar. 2015.

[3] Y. J. Zhang and J. Fan, "An intrinsic circuit model for multiple vias in an irregular plate pair through rigorous electromagnetic analysis," IEEE Trans. Microw. Theory Tech., vol. 58, no. 8, pp. 2251-2265, Aug. 2010.

[4] P. Li, L. J. Jiang and H. Bagci, "Transient analysis of dispersive powerground plate pairs with arbitrarily shaped antipads by the DGTD method with wave port excitation," IEEE Trans. Electromagn. Compat., vol. 59, no. 1, pp. 172-183, Sept. 2016. 